\title{
PC Configuration and Component Recommendation System
}

\author{
Someshkumar \\ Mishra \\ UG Student \\ Mumbai \\ India
}

\author{
Shreyas Bane \\ UG Student \\ Mumbai \\ India
}

\author{
Rahul Pandit \\ UG Student \\ Mumbai \\ India
}

\author{
Neelam Phadnis \\ Professor \\ Mumbai \\ India
}

\begin{abstract}
Recommendation Systems are created to recommend appropriate products to users from a collection of products with various complex attributes. Personal Computers have an assortment of components that vary incompatibility with each other. Due to the large number of possible configurations and parameters to consider, it is difficult for laymen to try making their configurations. It is also difficult to compare which components are similar to each other. This paper presents a recommendation system for these components using collaborative filtering.
\end{abstract}

\section{General Terms}

Machine Learning, KNN Algorithm, Collaborative Filtering, Recommendation System, Computer Hardware.

\section{Keywords}

PC Configuration, Recommendation, Machine Learning, KNN, Collaborative Filtering, Computer Hardware.

\section{INTRODUCTION}

In a world full of technologies, the number of products available to a person has drastically increased in amount and variety. Due to the large availability of products, choosing the right product is time-consuming. As a result, reducing the time taken for each decision is a major factor. Recommendation systems have become a valuable tool to save time. In a general way, recommender systems are algorithms aimed to suggest relevant items to users based on their interests or needs. Recommender systems are beneficial on the user side as well as the business side. On the business side, the company generates a huge amount of income when they are efficient or have a way to stand out significantly from competitors.PC product products have a large variety of components and not all users have sufficient knowledge about them to build their PCs from the components available to them. Incompatibility between components varies depending on specific factors. This introduces a steep learning curve for anyone who might be interested in making their PC config. This recommendation system is made to address this issue. It aims to allow users to select their components while checking the compatibility of the components for them while also recommending alternatives. It also allows users to get a PC config generated automatically for them based on their need for the PC. This significantly lowers the knowledge level required to create your PC config.

\section{LITERATURE REVIEW}

There have been many studies conducted on various types of recommendation systems. Developers create recommendation algorithms, and computers use those to determine conclusions based on the properties of the given set of data [1].There are mainly two types of recommendation systems, content based and collaborative. Content-based recommendation systems use the properties and description of an item and the user's rating of another set of items to estimate user's interest [2]. Collaborative filtering recommendation systems process information or patterns based on the collaboration of users or similarity between items[3].Collaborative filtering is better at prediction when compared to content-based filtering because it analyses and compares user's browsing history with other users and suggests results [4].

\section{PC CONFIGURATION AND COMPONENT RECOMMENDATION SYSTEM}

To allow users to create pc configurations a web app was created. Configurations are the combination of CPU, motherboard, ram, GPU, storage, and PSU in the PC. This web app allows users to create multiple pc configurations and store them.

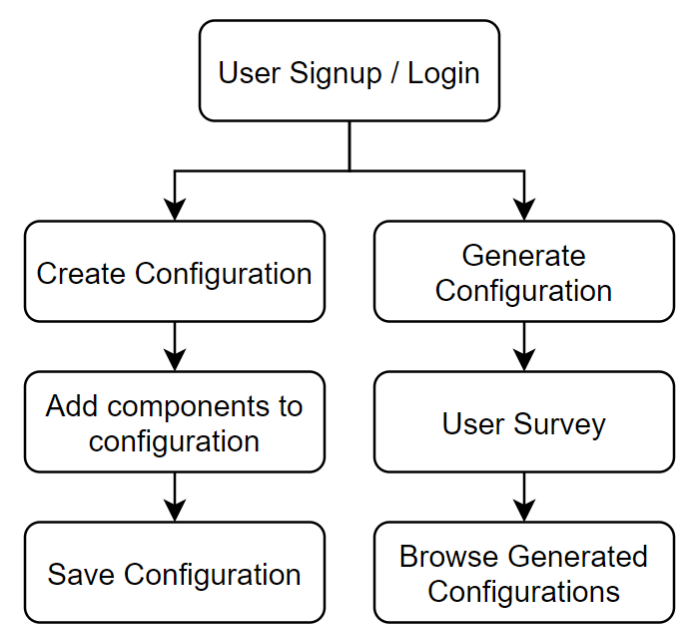

Figure 1: User Experience

As shown in Figure1, it also allows users to browse through the collection of components present, check out their specifications, and add these components to their builds. It also allows the users to have a configuration automatically created for them based on their answers to the questions presented to them.Component recommendations are presented to them while they are creating their pc configuration. While creating a configuration user has to add each component one by one into their configuration, one per category. While browsing for the component in a category the user is shown recommended components for that category. The user can select one of those or make their own independent choice. A 
compatibility check is done whenever a component is added to the configuration and the result is shown to the user. This is done to keep the user informed on whether the configuration is a valid one. The user can change the selected component at any time they want.Generating configuration automatically involves the surveying the user. This gives us an indication of what kind of pc configuration they want. A user who does not use their pc for heavier tasks does not need an expensive configuration. Once the demands of the user have been identified, multiple pc configurations satisfying those needs are presented to the user.All these options allow users of varying knowledge levels to create their pc configurations without being intimidated by the number of choices available, while also avoiding any common mistakes a layman might make.

\subsection{Checking for Compatibility}

Different types or categories of components have various attributes associated with them. It is usually possible to find out the compatibility between two different types of components by comparing certain attributes of these components. However, these comparisons are not valid for all possible cases due to decisions a manufacturer might make. These are edge cases and are very difficult to keep track of. The decision to ignore these was made because of their extreme rarity.This is a list of comparisons used to decide compatibility:

1. To find CPU compatibility, the socket type of the CPU must be the same as the socket type of the motherboard and the microarchitecture of the CPU must be supported by the motherboard chipset.

2. To find RAM compatibility, the RAM type must be the same as the Memory slot type on the motherboard and the RAM frequency must be present in the supported frequency ranges for the motherboard.

3. To find GPU compatibility, the GPU Interface must match at least one of the available expansion slots available on the motherboard.

4. To find Storage compatibility, the Storage interface must match at least one of the available connectors on the motherboard.

5. To find PSU compatibility, the PSU Wattage must always be higher than the total TDP of all the components present.

6. To find Motherboard compatibility, the above comparisons with different components must be valid.

If all of these conditions are satisfied then the configuration is compatible.

\subsection{Component Recommendation}

The component recommendation has two major steps.

1. Find the component for the selected category which has the highest number of occurrences in builds that are similar to the one being made.

2. Use KNN to find components similar to the above component and show them to the user along with the above component.

\subsection{Configuration Generation}

Configuration Generation has the following steps.

1. Based on the results of the user's survey their need is decided.

2. The top two of the most commonly used CPU appropriate for the user's need are selected.
3. For each of the CPUs, configs are created using the components which were most commonly paired with them.

4. KNN is used to create a list of components that are similar to components used in these two configs.

5. The components are combined into configs and are checked for compatibility.

6. The configs are then presented to the user along with the previous two configs as recommendations.

\subsection{K-nearest Neighbour (KNN)}

The KNN algorithm is a type of supervised machine learning algorithm that can be used for both classifications as well as regression for prediction problems.It has two main properties which as follows:

1. Lazy learning algorithm - $\mathrm{KNN}$ is considered a lazy learning algorithm because it does not have a specialized training phase and uses all the data for training while classification.

2. Non-parametric learning algorithm - KNN is considered a non-parametric learning algorithm because it doesn't assume anything about the underlying data.

It assumes that similar things exist in close to each other.

\subsubsection{Algorithm}

The steps are:

1. Load the data.

2. Initialize your $\mathrm{K}$ to the number of neighbours

3. The distance between the query example and the current example from the data is calculated.

4. The distance and the index of the example are added to an ordered collection.

5. Sort the ordered collection of distances and indices in ascending order of the distances.

6. Pick the first $\mathrm{K}$ entries from the sorted collections and get their labels. These are your most similar K-items.

\subsubsection{Distance Function}

For similarity, a distance function is needed for measurement between components with respect to its features value. For this, adjusted cosine similarity was used. The similarity between component a and component $\mathrm{b}$ can be calculated by the following formula.

$\operatorname{sim}(\mathrm{a}, \mathrm{b})=\frac{\sum_{u \in U(a) \cap U(b)}\left(P_{a, u}-\underline{P}_{u}\right)\left(P_{b, u}-\underline{P}_{u}\right)}{\sqrt{\sum_{u \in U(a) \cap U(b)}\left(P_{a, u}-\underline{P}_{u}\right)^{2} \sum_{u \in U(a) \cap U(b)}\left(P_{b, u}-\underline{P}_{u}\right)^{2}}}[3]$

Where,

$$
\begin{aligned}
& P_{a, u} \text { - feature value of feature u to product a. } \\
& P_{b, u} \text { - feature value of feature u to product } \mathrm{b} . \\
& \underline{P}_{u} \text { - average feature value of feature u. }
\end{aligned}
$$

\subsubsection{Component Prediction}

Select K neighbour components out of $\mathrm{N}$ components that have the highest similarity with the active components. These are our recommended components.

\section{DATA}

\subsection{Gathering and Formatting Data}

This recommendation system needs two types of data, component information, and user data. Component information is the data of all the individual components and their specifications, categorized into component types. An example of this is having a CPU table contain all the 


\begin{tabular}{|c|c|c|c|c|c|c|c|c|c|c|c|c|c|}
\hline 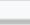 & A & 8 & c & D & E & $\mathrm{F}$ & o & H & 1 & ᄀ & $\mathrm{k}$ & м & $\mathrm{N}$ \\
\hline 1 & ID & Name & Manufacturer & Microarchitecture & Core Family & Core Count & No of Threads & Multithreading & Core Clock & Boost Clock & Socket & Integrated Graphics & Lithography \\
\hline 2 & C1 & Ryzen 31200 & AMD & Zen & Summit Ridge & & 4 & 4 NO & 3.1 & 3.4 & 65 AM4 & No & 14 \\
\hline 3 & C2 & Ryzen $31300 \mathrm{X}$ & AMD & Zen & Summit Ridge & & 4 & 4 NO & 3.5 & 3.7 & 65 AM4 & No & 14 \\
\hline 4 & C3 & Ryzen 51400 & AMD & Zen & Summit Ridge & & 4 & 8 YES & 32 & 3.4 & 65 AM4 & No & 14 \\
\hline 5 & C4 & Ryzen $51500 x$ & AMD & Zen & Summit Ridge & & 4 & 8 YES & 3.5 & 3.7 & 65 AM4 & No & 14 \\
\hline 6 & $\mathrm{C} 5$ & Ryzen 51600 & AMD & Zen & Summit Ridge & & 6 & 12 YES & 32 & 36 & 65 AM4 & No & 14 \\
\hline 7 & C6 & Ryzen $51600 x$ & AMD & Zen & Summit Ridge & & 6 & 12 YES & 3.6 & 4 & 95 AM4 & No & 14 \\
\hline 8 & $\mathrm{C7}$ & Ryzen 71700 & AMD & Zen & Summit Ridge & & 8 & 6 YES & 3 & 3.7 & 65 AM4 & No & 14 \\
\hline 9 & C8 & Ryzen $71700 \mathrm{x}$ & AMD & Zen & Summit Ridge & & 8 & 16 YES & 3.4 & 3.8 & 95 AM4 & No & 14 \\
\hline 10 & C9 & Ryzen 7 1800x & AMD & Zen & Summit Ridge & & 8 & 16 YES & 3.6 & 4 & 95 AM4 & No & 14 \\
\hline 11 & $\mathrm{C} 10$ & Ryzen Threadripper $1900 \mathrm{X}$ & AMD & Zen & Whitehaven & & 8 & 16 YES & 3.8 & 4 & 180 sTR4 & No & 14 \\
\hline 12 & $\mathrm{c} 11$ & Ryzen Threadripper 1920X & AMD & Zen & Whitehaven & & 12 & 24 YES & 3.5 & 4 & 180 sTR4 & No & 14 \\
\hline 13 & $\mathrm{C} 12$ & Ryzen Threadripper 1950X & AMD & Zen & Whitehaven & & 16 & 32 YES & 3.4 & 4 & 180 sTR4 & No & 14 \\
\hline 14 & C13 & Ryzen 322006 & AMD & Zen & Raven Ridge & & 4 & 4 NO & 3.5 & 3.7 & 65 AM4 4 & Radeon Vega 8 & 14 \\
\hline 15 & $\mathrm{C} 14$ & Ryzen $52400 \mathrm{G}$ & AMD & Zen & Raven Ridge & & 4 & 8 YES & 3.6 & 39 & 65 AM4 & Radeon Vega 11 & 14 \\
\hline 16 & C15 & Ryzen 31200 AF & AMD & Zent & Pinnacle Ridge & & 4 & 4 NO & 3.1 & 34 & 65 AM4 & No & 12 \\
\hline 17 & $\mathrm{C} 16$ & Ryzen 51600 AF & AMD & Zent & Pinnacle Ridge & & 6 & 12 YES & 32 & 3.6 & 65 AM4 & No & 12 \\
\hline 18 & c17 & Ryzen 52600 & AMD & Zent & Pinnacle Ridge & & 6 & 12 YES & 3.4 & 39 & 65 AM4 & No & 12 \\
\hline 19 & $\mathrm{C} 18$ & Ryzen $52600 x$ & AMD & Zent & Pinnacle Ridge & & 6 & 12 YES & 3.6 & 4.2 & 95 AM4 & No & 12 \\
\hline 20 & $\mathrm{C} 19$ & Ryzen 72700 & AMD & Zent & Pinnacle Ridge & & 8 & 16 YES & 32 & 4.1 & 65 AM4 & No & 12 \\
\hline 21 & $\mathrm{C} 20$ & Ryzen $72700 x$ & AMD & Zent & Pinnacle Ridge & & 8 & 6 YES & 3.7 & 4.3 & 105 AM4 & No & 12 \\
\hline 22 & $\mathrm{C} 21$ & Ryzen Threadripper 2920X & AMD & Zent & Colfax & & 12 & 24 YES & 3.5 & 43 & 180 sTR4 & No & 12 \\
\hline 23 & $\mathrm{C} 22$ & Ryzen Threadinpper 2950X & AMD & Zent & Colfax & & 16 & 32 YES & 3.5 & 44 & 180 sTR4 & No & 12 \\
\hline 24 & $\mathrm{C} 23$ & Ryzen Threadripper $2970 \mathrm{WX}$ & AMD & Zent & Colfax & & 24 & 18 YES & 3 & 4.2 & 250 sTR4 & No & 12 \\
\hline 25 & $\mathrm{C} 24$ & Ryzen Threadripper $2990 \mathrm{Wx}$ & AMD & Zent & Colfax & & 32 & 34 YES & 3 & 4.2 & 250 sTR4 & & 12 \\
\hline 26 & $\mathrm{C} 25$ & Rvzen $33200 \mathrm{G}$ & AMD & Zent & Picasso & & 4 & 4 NO & 3.6 & 4 & 65 AM4 & Radeon Veaa 8 & 12 \\
\hline
\end{tabular}

Figure 2: CPU Data

specifications as individual columns and each CPU being its row. The categories of the components are processors (CPU), motherboards, volatile memory (RAM), graphic card (GPU), permanent storage, power supplies (PSU). Data of all these components were collected from various manufacturer and vendor websites using web scraping and compiled into CSV sheets. Components are also tagged according to the price range they fall under. Figure 2 shows a snippet of data for the CPU category.User data is the collection of PC configs made by other users. It contains individual configs as rows and component categories as columns. For example, each row will have a Config ID, User ID, CPU ID, Motherboard ID, RAM ID, GPU ID, Storage ID, and PSU ID in the individual columns. All the configs have compatible components in them.

\subsection{Data Pre-processing}

The component dataset contains required independent categorical values. The recommendation system only accepts numeric data for component attributes. To satisfy this need, some attributes were converted from text to integers by giving them values based on how functionally different they are from each other. An example of this would be the form factor of the motherboards. It ranges from small Mini ITX boards to large EATX boards. These were converted to an integer starting from 1 for Mini ITX to an increment by 1 for each increment in size, turning them into measurable values for the recommendation system to use.

\subsection{Limitations}

It was not possible to gather the required user data at a large scale and in the format required without surveying a large group of people. Due to the lack of resources and special circumstances, conducting such a survey was not possible. To address this issue, a simulated dataset was created that represents real-world data in a satisfactory manner. This was done for testing purposes. Therefore, it should be noted that the recommendations might not be satisfactory for real-world purposes and are influenced by the biases present in our data. This issue can be solved by collecting real-world data and feeding it to the system.Component data used also does not include all the products released by manufacturers for each category. Also, only the components manufactured from 2017 - 2021 were included due to older components usually being discontinued by the manufacturers.

\section{APPLICATION}

Due to the way PC configurations can be created easily using this, the recommendation system can be used by people who want to experiment with creating PC configs. The knowledge about PC components required to create a PC configuration is also very high. This can be used as a teaching tool to teach people about PC building and configuration.E - vendors of PC components can use this to improve customer interaction and even sales through recommendation. Creating configurations allows customers to get more exposure to the component, increasing their chances of buying it. User data can also be collected for data analysis.

\section{RESULTS}

\subsection{Recommendation System}

The KNN algorithm when given the dataset of the component and the component that was selected the most gives the results indicating what components from that component's category are similar to it. Figure 3 shows the formatted output of the algorithm with $\mathrm{G} 1$ and the other similar numbers being the GPU ID.

Recommendations for $\mathrm{G} 1$ :

1: G1, with distance of 0.0 :

2: G3, with distance of $\theta . \theta$ :

3: G4, with distance of 0.0010509332288555218 :

4: G22, with distance of 0.10303978373685219 :

5: G23, with distance of 0.10303978373685219 :

6: G21, with distance of 0.10303978373685219 :

\section{Figure 3:Formatted KNN Output}

\subsection{Web App / GUI of the Recommendation System}

The implemented web app allows users to create PC configs. Figure4 shows how each component can be added. Figure5 shows how components are recommended while adding them. Configs can also be automatically generated and viewed by the user. Figure6 shows how generated configs are shown. 


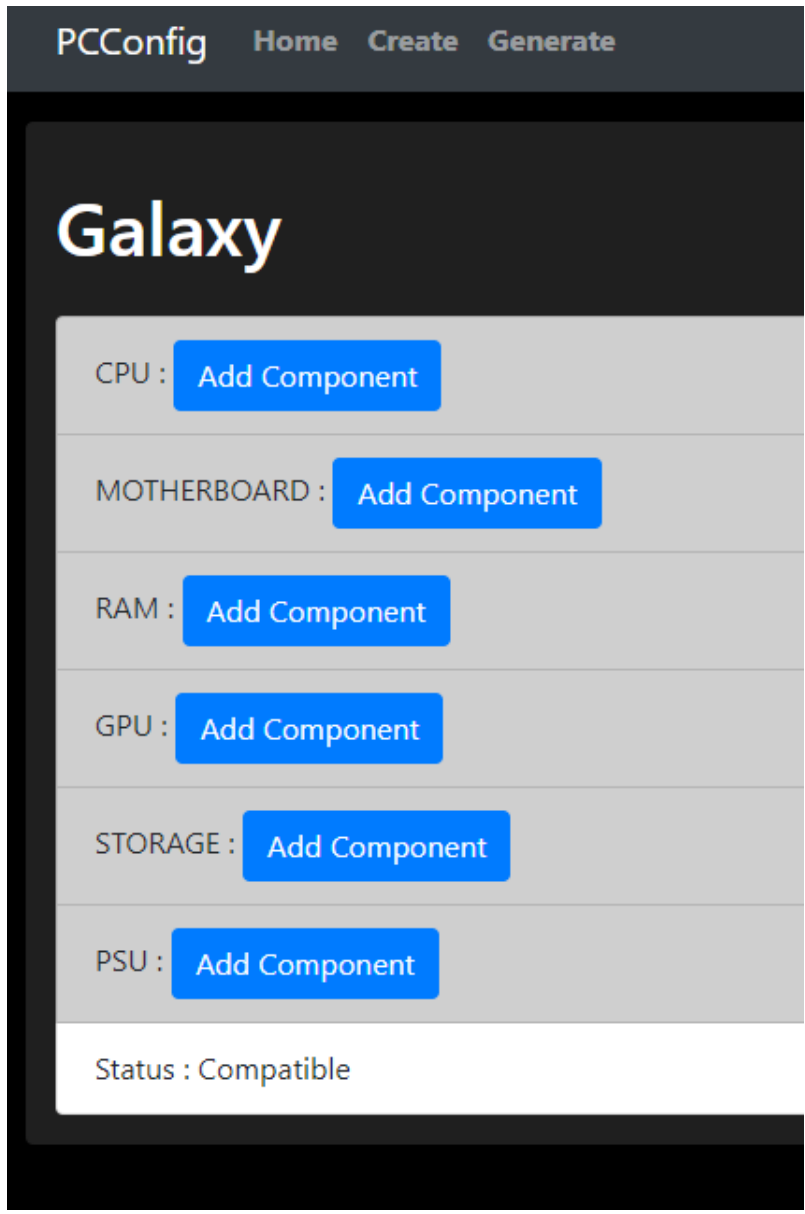

Figure 4:PC Configuration

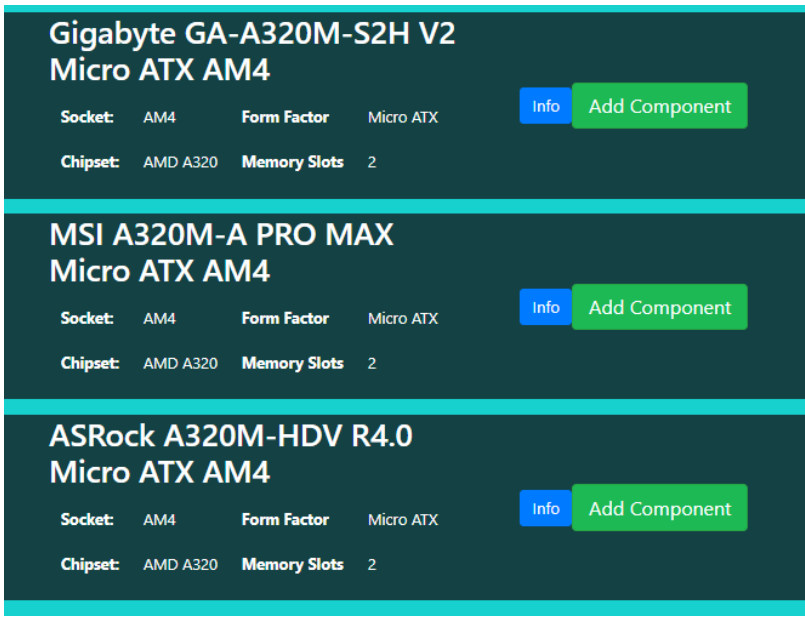

Figure 5: Getting Recommendation
Womble

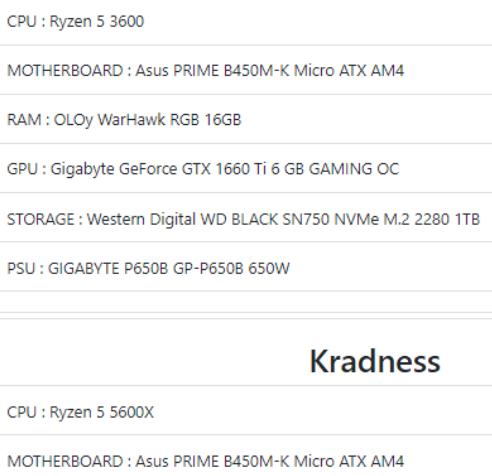

Figure 6: Generated Configurations

\section{CONCLUSION AND FUTURE WORK}

Following things can be done in the future to improve the system:

1. Using different machine learning algorithms for the recommendation. Using Deep Learning for creating a recommendation system capable of interacting with more attributes automatically. Using weighted attributes, allowing us to get more precise recommendations.

2. Gathering pricing data of the components and using it for improving recommendations.

3. Improving our dataset by adding more components and attributes, also including accessories and not just core components used in a PC.

4. Doing surveys to get real-world data and to gather statistical data for the results of the recommendation.

\section{ACKNOWLEDGMENT}

The timely completion of the project 'PC Configuration and Component Recommendation System' has been possible because of our teacher and project guide Prof. Neelam Phadnis who provided us with guidance and motivation throughout its making. We thank her for giving us an opportunity to create this project.

\section{REFERENCES}

[1] Nawrocka, A. Kot and M. Nawrocki, "Application of machine learning in recommendation systems," 2018 19th International Carpathian Control Conference (ICCC), Szilvasvarad, 2018, pp. 328-331, doi: 10.1109/CarpathianCC.2018.8399650.

[2] Zisopoulos, Charilaos\&Karagiannidis, Savvas\&Demirtsoglou, Georgios \&Antaris, Stefanos. (2008). Content-Based Recommendation Systems.

[3] Wen, Zheng. (2012). Recommendation System Based on Collaborative Filtering.

[4] M. Gupta, A. Thakkar, Aashish, V. Gupta and D. P. S. Rathore, "Movie Recommender System Using Collaborative Filtering," 2020 International Conference on Electronics and Sustainable Communication Systems (ICESC), Coimbatore, India, 2020, pp. 415-420, doi: 10.1109/ICESC48915.2020.915 